\title{
Ni-Zn Ferrite-graphene Nanohybrids: Synthesis and Characterization of Magnetic and Microwave Absorbing Properties
}

\author{
Yau Thim $\mathrm{Ng}^{1}$, Wei Kong ${ }^{2}$, Kim Yeow Tshai $^{1}$ and Ing Kong ${ }^{1, \text { a }}$ \\ ${ }^{1}$ Department of Mechanical, Materials and Manufacturing Engineering, The University of Nottingham Malaysia, Jalan Broga 43500 Semenyih, \\ Selangor, Malaysia \\ ${ }^{2}$ Faculty of Applied Science and Foundation Studies, Infrastructure University Kuala Lumpur Unipark Suria, Jalan Ikram-Uniten, 43000 Kajang, \\ Selangor, Malaysia.
}

\begin{abstract}
An in-situ deposition technique was used in the synthesis of Ni-Zn ferrite-graphene (NZFG) nanohybrids. The XRD patterns revealed the presence of cubic spinel structure of Ni-Zn ferrite (NZF) nanoparticles with good crystallinity and small crystallite sizes. The SEM images showed NZF nanoparticles were uniformly deposited on graphene sheets. The effect of different loading amounts of NZF nanoparticles in the nanohybrids was also investigated by tuning the mass ratio of $\mathrm{FeCl}_{3}$ and expanded graphite (EG). The magnetic measurements showed ferromagnetic behaviour with low coercivity. Improvements in saturation magnetization of the nanohybrids can be seen with increasing mass ratio of $\mathrm{FeCl}_{3}$ : EG. The microwave absorption properties were determined based on the measured relative complex permittivity and permeability. For the nanohybrids, the minimum reflection loss $\left(\mathrm{R}_{\mathrm{L}}\right)$ obtained is $-37.57 \mathrm{~dB}$ at $7.54 \mathrm{GHz}$ and the absorbing bandwidth in which the $\mathrm{R}_{\mathrm{L}}$ is less than -10 $\mathrm{dB}$ is $7.30 \mathrm{GHz}$ when the NZF content was $79 \mathrm{wt} \%$ at $7 \mathrm{~mm}$ thickness. The enhancement in the minimum $\mathrm{R}_{\mathrm{L}}$ was due to the synergistic effect between NZF nanoparticles and graphene.
\end{abstract}

\section{Introduction}

In recent years, multifunctional nanocomposites comprised of graphene and magnetic nanoparticles have gained a significant amount of interest due to their combinations of properties that are potentially useful in a variety of applications [1,2]. Graphene, a wonder material having extraordinary electrical, thermal and mechanical properties [3], possesses large surface areas which can be used as a template for the attachment of magnetic nanoparticles.

$\mathrm{Ni}-\mathrm{Zn}$ ferrite (NZF) is considered an excellent candidate of magnetic materials due to their high saturation magnetization, high electrical resistivity, good mechanical strength and chemical stability [4]. In terms of their synthesis methods, chemical methods are more advantageous over traditional milling methods in the preparation of ferrites due to its precise compositions, higher degree of homogeneity and better control of nanoparticle size. In particular, chemical co-precipitation is considered the most convenient method due to its simplicity and better control over the crystallite size and other properties of the materials. However, in many cases subsequent heating and annealing of the material is required to form the final product [4-7]. In light of works by [8-10], it has become possible to synthesize ferrites at room temperature.

In the present work, Ni-Zn ferrite-graphene (NZFG) nanohybrids with a Ni-Zn ferrite (NZF) composition of $\mathrm{Ni}_{0.5} \mathrm{Zn}_{0.5} \mathrm{Fe}_{2} \mathrm{O}_{4}$ were successfully synthesized through a facile in-situ deposition method at room temperature. The graphene sheets were produced by ultrasonication of expanded graphite (EG). The mass ratio of $\mathrm{FeCl}_{3}$ to $\mathrm{EG}$ was varied from 5:1, 10:1 to 20:1 giving three different samples denoted as NZF5G1, NZF10G1 and NZF20G1. The microstructure, morphology and magnetic properties of the resulting nanohybrids were reported.

\section{Experimental}

\subsection{Preparation of Ni-Zn ferrite graphene (NZFG) nanohybrids}

NZFG nanohybrids were prepared by an in-situ deposition method involving the co-precipitation of NZF nanoparticles on graphene. EG was ultrasonicated in $20 \mathrm{ml}$ deionized water for $30 \mathrm{~min}$ to produce a graphene suspension. For NZF composition $\left(\mathrm{Ni}_{0.5} \mathrm{Zn}_{0.5} \mathrm{Fe}_{2} \mathrm{O}_{4}\right), \mathrm{NiCl}_{2} \cdot 6 \mathrm{H}_{2} \mathrm{O}, \mathrm{ZnCl}_{2}, \mathrm{FeCl}_{2} \cdot 4 \mathrm{H}_{2} \mathrm{O}$ and $\mathrm{FeCl}_{3} \cdot 6 \mathrm{H}_{2} \mathrm{O}$ with molar ratio 1:1:2:2 were dissolved into $180 \mathrm{ml}$ deionized water under nitrogen protection for $30 \mathrm{~min}$. The graphene suspension was added to the solution of NZF at three different mass ratio of $\mathrm{FeCl}_{3}: \mathrm{EG}(5: 1,10: 1$ and 20:1) and stirred for a further $15 \mathrm{~min}$. With vigorous stirring, $\mathrm{NaOH}$ solution was added to the mixture dropwise to maintain $\mathrm{pH}$ of 10-11. The reaction was carried out for $30 \mathrm{~min}$. The suspension was then repeatedly washed

\footnotetext{
${ }^{a}$ Corresponding author : ing.kong@nottingham.edu.my
} 
until $\mathrm{pH} \sim 7$, filtered, dried at $60^{\circ} \mathrm{C}$ in air for 24 hours. In the absence of graphene, the NZF nanoparticles were obtained via the same procedure for comparison.

\subsection{Characterization}

X-ray diffraction (XRD) was conducted using a Bruker D8 Advance system based on reflectance operated at $45 \mathrm{kV}$ and $35 \mathrm{~mA}$ with $\mathrm{CuK} \alpha_{1}$ radiation $(\lambda=1.54059 \AA)$ in the $2 \theta$ range $10-80^{\circ}$, in steps of $0.04^{\circ}$. The morphology of the samples at different magnifications was observed by field emission scanning electron microscopy (FESEM) equipment Quanta400F (FEI), conducted in low vacuum mode, with an accelerating potential of $200 \mathrm{~V}-30 \mathrm{kV}$. The magnetic properties of the hybrids were studied using vibrating sample magnetometer (VSM), model Lakeshore 7404 Series at room temperatures $\left(25{ }^{\circ} \mathrm{C}\right)$. The measurements were carried out in a maximum field of $12 \mathrm{kOe}$. Magnetic parameters such as saturation magnetization $\left(\mathrm{M}_{\mathrm{S}}\right)$, remanence $\left(\mathrm{M}_{\mathrm{R}}\right)$, and coercivity $\left(\mathrm{H}_{\mathrm{C}}\right)$ were determined. For microwave absorbing properties, specimens were prepared by uniformly mixing $30 \mathrm{wt} \cdot \%$ NZF and NZFG nanohybrids with paraffin wax at $70{ }^{\circ} \mathrm{C}$ and pressed into toroidal shape with inner diameter of $2.9 \mathrm{~mm}$ and outer diameter of $6.9 \mathrm{~mm}$. The microwave absorption parameters of complex permittivity and complex permeability were measured at the frequency range 1-18 GHz by transmission/reflection method using an Anritsu 37247D microwave vector network analyzer with GPC-7 mm coaxial line.

\section{Results and Discussion}

The microstructure of NZF and NZFG nanohybrids were analysed by XRD technique and is shown in Fig. 1. All the diffraction patterns show broad peaks which indicate particles of fine crystallite sizes. NZF nanoparticles synthesized in the absence of graphene show characteristic peaks at $2 \theta=30.18^{\circ}, 35.43^{\circ}, 43.11^{\circ}, 52.15^{\circ}, 56.90^{\circ}$, and $62.59^{\circ}$ that can be assigned to $(220)$, $(311)$, (400), (422), (511) and (440) lattice planes of cubic unit cell of ferrite. These values match the single crystalline spinel form of the Ni-Zn ferrite (JCPDS Card No. 8-234). In all the Ni-Zn ferrite-graphene nanohybrids, the peaks of NZF appeared intense and are matched well, which confirms that NZF nanoparticles were successfully deposited on graphene. The diffraction peak at $2 \theta=26.5^{\circ}$ corresponds to the (002) plane of graphene. An additional peak which appears at $2 \theta=64.4^{\circ}$ (marked by *) shows the presence of $\alpha-\mathrm{Fe}_{2} \mathrm{O}_{3}$ phase impurity. According to [11,12], this impurity exists when low amounts of precipitating agent were used. The average crystallite size based on Scherrer's formula (Eq. 1) were estimated to be $12.47 \mathrm{~nm}, 8.72 \mathrm{~nm}, 10.27 \mathrm{~nm}$ and $13.43 \mathrm{~nm}$ for NZF, NZF5G1, NZF10G1 and NZF20G1, respectively. The results show that the average crystallite size for NZFG nanohybrids increases with the mass ratio of $\mathrm{FeCl}_{3}$ :EG.

$$
D=0.89 \lambda / \beta \cos \theta
$$

where $\mathrm{D}$ is the crystallite size, $\lambda$ is the X-ray wavelength, $\theta$ is the diffraction angle and $\beta$ is the half width of the observed diffraction peak.

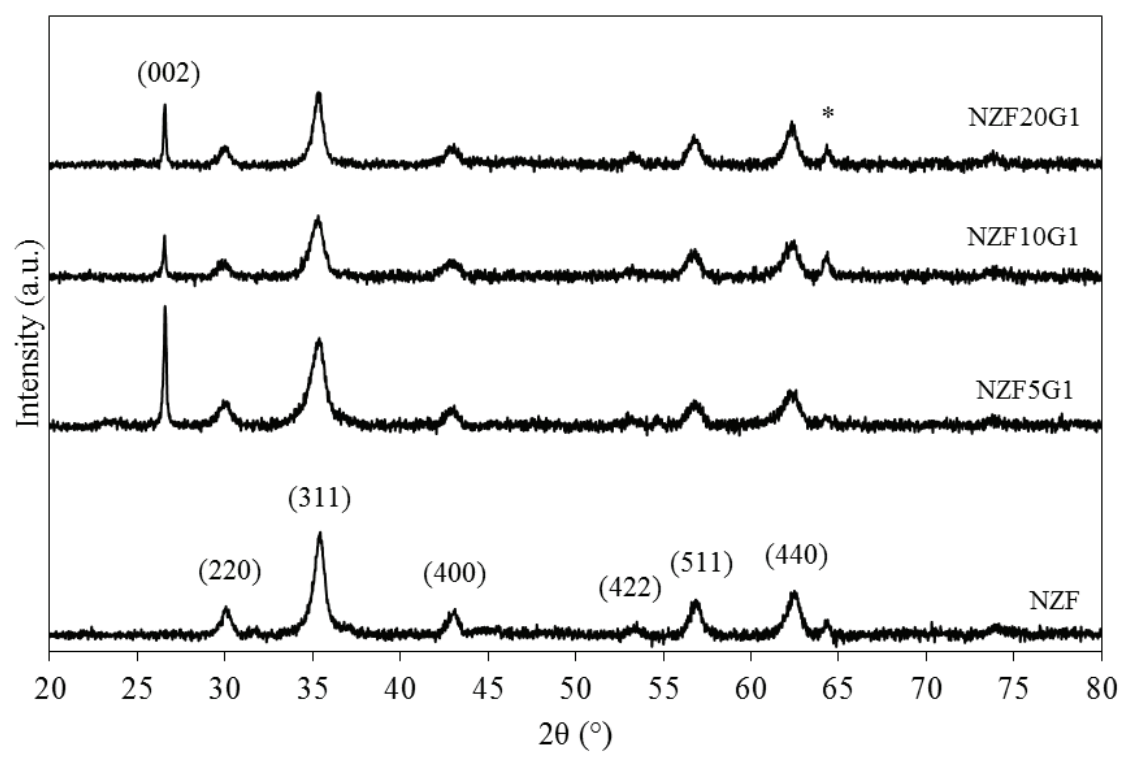

Figure 1. X-ray diffractograms of NZF, NZF5G1, NZF10G1 and NZF20G1 

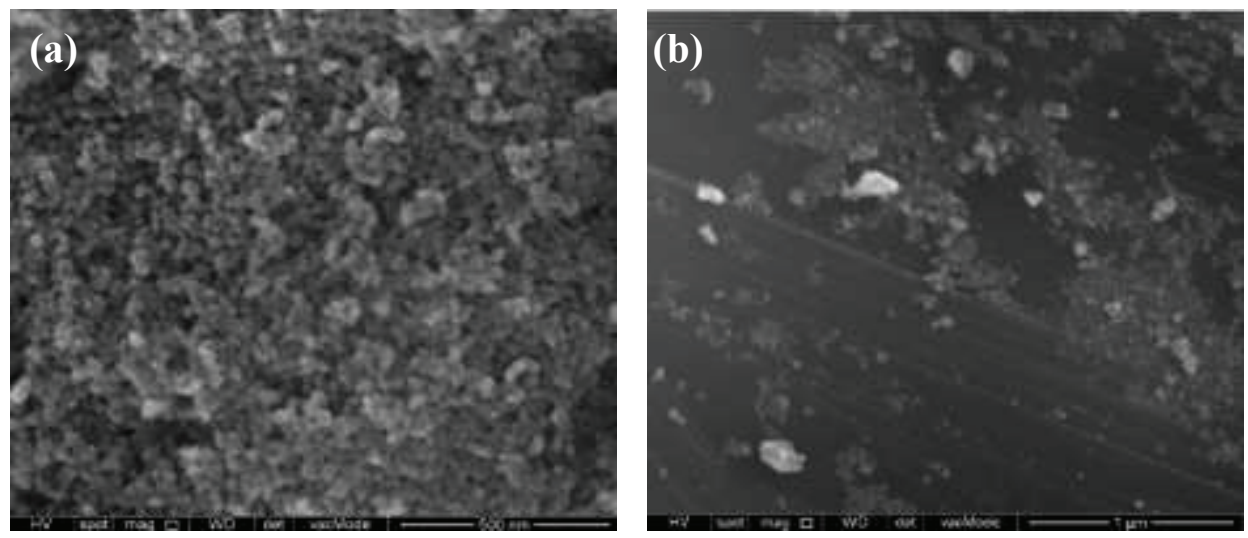

Figure 2. SEM images of (a) NZF and (b) NZF10G1.

Fig. 2 shows the SEM images of NZF and NZFG nanohybrids. It can be seen in Fig. 2(a) that the as-synthesized NZF nanoparticles have taken a spherical shape. As for NZFG nanohybrids, Fig. 2(b) shows that NZF nanoparticles were densely distributed over the surfaces of graphene sheets. The images indicate success in the formation of NZF in the presence of graphene.

The magnetization curves of NZF and NZFG nanohybrids are shown in Fig. 3. The values of magnetic measurements were determined from hysteresis loops and were summarized in Table 1 . NZF nanoparticles recorded the highest $\mathrm{M}_{\mathrm{S}}$ of 42.50 emu/g, which is higher than that produced in $[5,13]$ but lower than that produced in [9]. A thin hysteresis was observed, showing the ferromagnetism of the nanoparticles at room temperature. As for $\mathrm{NZFG}, \mathrm{M}_{\mathrm{S}}$ values increase along with the mass ratio of $\mathrm{FeCl}_{3}$ to EG. Due to the non-magnetic nature of graphene, the increment in $\mathrm{M}_{\mathrm{S}}$ of the nanohybrids is likely driven by the amount and size effect of the NZF nanoparticles. In terms of size effect, larger sized nanoparticles tend to have higher $\mathrm{M}_{\mathrm{S}}$ due to lesser surface distortions and magnetocrystalline anisotropy distortions of the nanoparticles [14]. Compared to $M_{S}$, relatively $s_{\text {aller }} M_{R}$ values for the samples implies that most of the spin moments has returned to their original state.

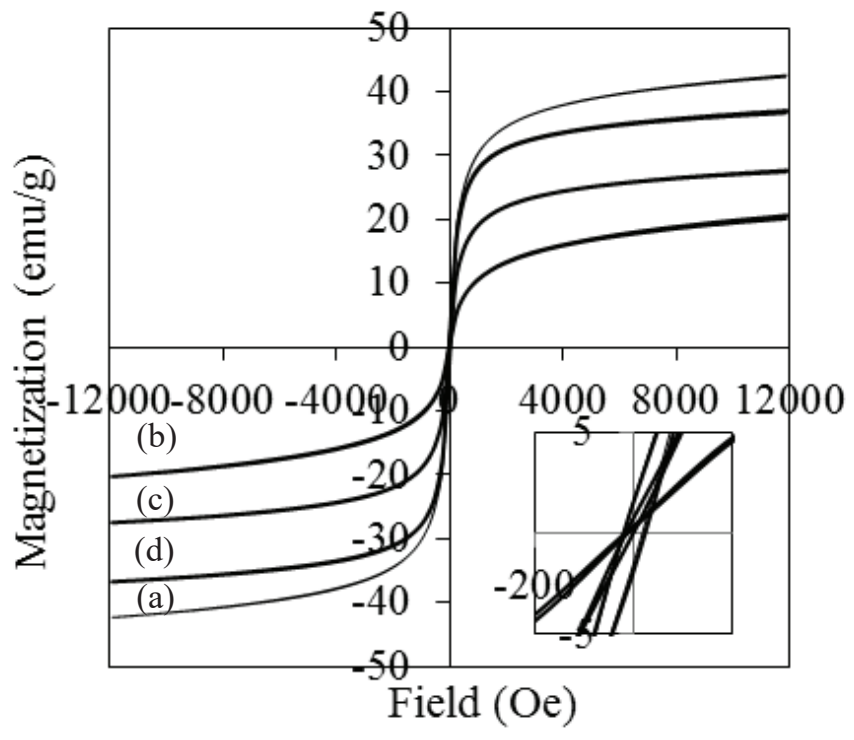

Figure 3. Magnetization curves of (a) NZF, (b) NZF5G1, (c) NZF10G1 and (d) NZF20G1.

Table 1. Magnetization characteristics of NZF, NZF5G1, NZF10G1 and NZF20G1.

\begin{tabular}{|c|c|c|c|}
\hline Sample & $\mathbf{M}_{\mathbf{S}}(\mathbf{e m u} / \mathbf{g})$ & $\mathbf{M}_{\mathbf{R}}(\mathbf{e m u} / \mathbf{g})$ & $\mathbf{H}_{\mathbf{C}}(\mathbf{O e})$ \\
\hline NZF & 42.50 & 1.47 & 17.92 \\
\hline NZF5G1 & 20.46 & 0.25 & 11.14 \\
\hline NZF10G1 & 27.60 & 0.66 & 13.89 \\
\hline NZF20G1 & 36.90 & 1.51 & 19.19 \\
\hline
\end{tabular}

The microwave absorption properties of wax composites containing $30 \mathrm{wt} \cdot \% \mathrm{NZF}$ and NZFG nanohybrids (NZF5G1, NZF10G1, NZF20G1) were determined by its complex permittivity $\left(\varepsilon_{\mathrm{r}}=\varepsilon^{\prime}-\mathrm{j} \varepsilon^{\prime \prime}\right)$ and complex permeability $\left(\mu_{\mathrm{r}}=\mu{ }^{\prime}-\mathrm{j} \mu\right.$ ”), which can be measured using a vector network analyzer. Based on the measured microwave parameters, the reflection loss $\left(R_{L}\right)$ can be calculated according to the transmission line theory using the two formulas below: 


$$
\mathrm{R}_{\mathrm{L}}=20 \log \left|\frac{\left(\mathrm{Z}_{\text {in }}-\mathrm{Z}_{0}\right)}{\left(\mathrm{Z}_{\text {in }}+\mathrm{Z}_{0}\right)}\right|
$$

where $R_{L}$ denotes the reflection loss in decibels $(d B), Z_{0}$ is the characteristic impedance of free space/air $(377 \Omega), Z_{\text {in }}$ is the input characteristic impedance of absorber. $Z_{\text {in }}$ can be expressed as:

$$
\mathrm{Z}_{\text {in }}=\mathrm{Z}_{0}\left(\frac{\mu_{\mathrm{r}}}{\varepsilon_{\mathrm{r}}}\right)^{1 / 2} \tanh \left\{\mathrm{j}\left(\frac{2 \pi \mathrm{fd}}{\mathrm{c}}\right)\left(\mu_{\mathrm{r}} \varepsilon_{\mathrm{r}}\right)^{1 / 2}\right\}
$$

where $\varepsilon_{\mathrm{r}}$ and $\mu_{\mathrm{r}}$ are the measured relative complex permittivity and permeability, $\mathrm{c}$ is the velocity of light, $\mathrm{f}$ is the frequency of the microwave and $d$ is the thickness of absorber.

Fig. 4 shows the calculated $R_{L}$ of NZF and NZFG nanohybrids with a thickness of $7 \mathrm{~mm}$. It can be seen that the $R_{L}$ for NZF reaches a minimum of $-31.56 \mathrm{~dB}$ at $7.33 \mathrm{GHz}$. As for NZFG nanohybrids, varying the NZF content varied the maximum absorption and position of the dips of the minimum $\mathrm{R}_{\mathrm{L}}$. First the minimum $\mathrm{R}_{\mathrm{L}}$ increases from $-30.65 \mathrm{~dB}$ at $7.15 \mathrm{GHz}$ to $-37.57 \mathrm{~dB}$ at $7.54 \mathrm{GHz}$ and then decreases to $-27.42 \mathrm{~dB}$ at $7.24 \mathrm{GHz}$. It is noteworthy that the frequency related to the $\mathrm{R}_{\mathrm{L}}$ minimum lies within the C-band $(4-8 \mathrm{GHz})$ of the electromagnetic spectrum, which is normally used for satellite communications. The bandwidth corresponding to $\mathrm{R}_{\mathrm{L}}$ below $-10 \mathrm{~dB}$ for NZF5G1, NZF10G1 and NZF20G1 are 8.00, 7.30 and $7.00 \mathrm{GHz}$, respectively. Based on the results, the enhancement in the microwave absorption properties for can be related to the synergistic effects of graphene coupled with NZF nanoparticles. When the dielectric and magnetic characteristics are matched well as in the case of NZF10G1, maximum absorption of the microwave energy occurs.

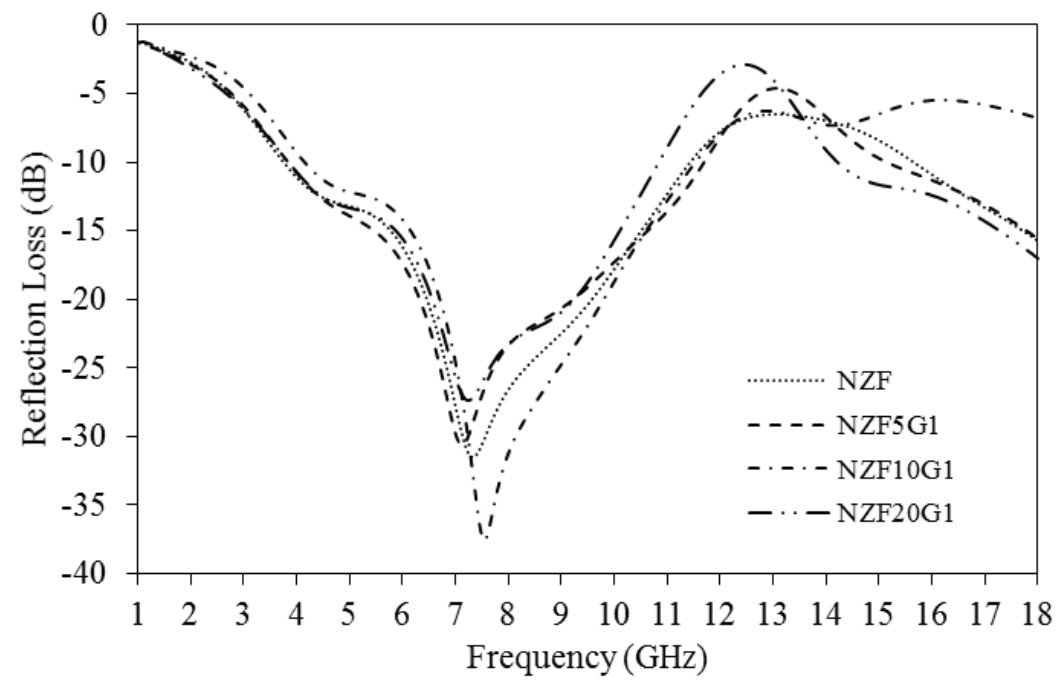

Figure 4. Frequency dependence of reflection loss for NZF and NZFG nanohybrids

\section{Conclusion}

In conclusion, a facile method was demonstrated to synthesis Ni-Zn ferrite-graphene (NZFG) nanohybrids. This method is capable of producing fine crystallite size nanoparticles with good crystallinity. The nanohybrids also showed good magnetic properties owing to a good distribution of NZF nanoparticles over the graphene sheets. Enhancement in the microwave absorption properties due to synergistic effect between NZF nanoparticles and graphene was seen when the NZF content in the nanohybrids is $79 \mathrm{wt} . \%$. It is anticipated that these nanohybrids can be used as fillers in developing multifunctional polymer composites.

\section{References}

1. C. Wang, C. Feng, Y. Gao, X. Ma, Q. Wu, Z. Wang, Chem. Eng. J. 173, $92-97$ (2011)

2. Y. Fu, Q. Chen, M. He, Y. Wan, X. Sun, H. Xia, et al., Ind. Eng. Chem. Res. 51, 11700-11709 (2012)

3. A.K. Geim, Science 324, 1530-1534 (2009)

4. I.H. Gul, W. Ahmed, A. Maqsood, J. Magn. Magn. Mater. 320, 270-275 (2008)

5. G.S. Shahane, A. Kumar, M. Arora, R.P. Pant, K. Lal, J. Magn. Magn. Mater. 322, 1015-1019 (2010)

6. A.S. Albuquerque, J.D. Ardisson, W.A.A. Macedo, M.C.M. Alves, J. Appl. Phys. 87, 4352 (2000)

7. S.M. Olhero, D. Soma, V.S. Amaral, T.W. Button, F.J. Alves, J.M.F. Ferreira, J. Eur. Ceram. Soc. 32, 2469-2476 (2012)

8. K. Nishimura, M. Abe, M. Inoue, IEEE Trans. Magn. 38, 3222-3224 (2002)

9. X. Lu, G. Liang, Q. Sun, C. Yang, Mater. Lett. 65, 674-676 (2011)

10. O. Perales Perez, K. Tohji, Y. Umetsu, J. Alloys Compd. 290, 129-136 (1999)

11. S. Thakur, S.C. Katyal, M. Singh, J. Magn. Magn. Mater. 321, 1-7 (2009)

12. K. Gandha, K. Elkins, N. Poudyal, J. Ping Liu, J. Appl. Phys. 117, 17A736 (2015)

13. B.P. Rao, A.M. Kumar, K.H. Rao, Y.L.N. Murthy, O.F. Caltun, I. Dumitru, et al., Adv. Mater. 8, 1703-1705 (2006)

14. C. Jiang, R. Liu, X. Shen, L. Zhu, F. Song, Powder Technol. 211, 90-94 (2011) 